\title{
Neutron production from thick LiF, C, Si, Ni, Mo, and Ta targets bombarded by 13.4-MeV deuterons
}

\author{
Hayato Takeshita ${ }^{1, *}$, Yukinobu Watanabe ${ }^{1}$, Keita Nakano $^{1}$, Seiya Manabe ${ }^{1}$, Katsumi Aoki ${ }^{1}$, Naoto Araki $^{1}$, \\ Kosuke Yoshinami ${ }^{1}$, Tadahiro Kin ${ }^{1}$, Nobuhiro Shigyo $^{2}$, Jun $\mathrm{Koga}^{3}$, So Makise ${ }^{3}$, Tamaki Yoshioka ${ }^{4}$, \\ Masaomi Tanaka ${ }^{5}$, and Takashi Teranishi ${ }^{3}$ \\ ${ }^{1}$ Department of Advanced Energy Engineering Science, Kyushu University, Fukuoka 816-8580 Japan \\ ${ }^{2}$ Department of Applied Quantum Physics and Nuclear Engineering, Kyushu University, Fukuoka 819-0395, Japan \\ ${ }^{3}$ Department of Physics, Kyushu University, Fukuoka 819-0395, Japan \\ ${ }^{4}$ Research Center for Advanced Particle Physics, Kyushu University, Fukuoka 819-0395, Japan \\ ${ }^{5}$ Research Center for Superheavy Elements, Kyushu University, Fukuoka 819-0395, Japan
}

\begin{abstract}
Double-differential thick target neutron yields from LiF, C, Si, Ni, Mo, and Ta targets bombarded by 13.4-MeV deuterons were measured by using an EJ-301 liquid organic scintillator at the Center for Accelerator and Beam Applied Science, Kyushu University. The measured $(d, x n)$ spectra were compared with the $(t, x n)$ spectra measured by the other group at the same incident energy per nucleon $(6.7 \mathrm{MeV} / \mathrm{u})$ and theoretical model calculations by Particle and Heavy Ion Transport code System (PHITS) and DEUteron-induced Reaction Analysis Code System (DEURACS). Some bumps are observed in the $(d, x n)$ spectra for low-Z target elements, while no specific structure was seen in the $(t, x n)$ spectra. The PHITS calculation, in which the intra-nuclear cascade of Liége (INCL) and generalized evaporation model (GEM) were used, generally overestimates neutron spectra while the DEURACS calculation agrees with experimental ones fairly well.
\end{abstract}

\section{Introduction}

As a candidate for intense neutron sources for nuclear applications, accelerator-based neutron sources using deuteron-induced reactions have been proposed in recent years. For example, at the International Fusion Materials Irradiation Facility (IFMIF), neutron beams generated by the $\operatorname{Li}(d, x n)$ reactions will be utilized in various irradiation tests for research and development of fusion reactors [1]. In these neutron sources, systematic estimation of neutron yields from the whole neutron source including accelerator is important because neutrons are produced not only from neutron converters made of light nuclei such as $\mathrm{Li}, \mathrm{Be}$, and $\mathrm{C}$ but also from structural materials of the accelerator. Therefore, we have measured systematically deuteron-induced thick target neutron yields (d-TTNYs) from various target materials [2], and validation of theoretical model calculation codes such as Particle and Heavy Ion Transport code System (PHITS) [3] and DEUteron-induced Reaction Analysis Code System (DEURACS) [4] has been performed.

On the other hand, Drosg et al. [5] have recently tackled the neutron production with triton irradiation, and measured the triton-induced thick target neutron yields (t-TTNYs) from various target materials using 6.7 MeV/u triton beams. Comparing the $(t, x n)$ and $(d, x n)$ data might offer better understanding of the nuclear reaction mechanism.

*e-mail: takeshita.hayato.890@s.kyushu-u.ac.jp
In this work, we measured d-TTNYs at an incident energy of $13.4 \mathrm{MeV}(6.7 \mathrm{MeV} / \mathrm{u})$ to investigate the difference between the $(d, x n)$ and $(t, x n)$ spectra from the same materials ( $\mathrm{LiF}, \mathrm{Si}, \mathrm{Ni}, \mathrm{Mo}$, and $\mathrm{Ta}$ ). Moreover, we validated the theoretical nuclear reaction models implemented in PHITS and DEURACS by comparing their predictions with the measured $(d, x n)$ spectra.

\section{Experimental Procedure}

The experiment was performed at the Center for Accelerator and Beam Applied Science (CABAS), Kyushu University. In the experiment, C, LiF, Si, Ni, Mo, and Ta were employed as the target materials. Figure 1 illustrates the experimental setup. Each target was placed in a vacuum chamber and bombarded by the $13.4-\mathrm{MeV}$ deuterons accelerated by an 8-MV Tandem Van de Graaf accelerator. The targets were thick enough to stop incident deuterons completely ( $2 \mathrm{~mm}$ for $\mathrm{C}, 1 \mathrm{~mm}$ for $\mathrm{LiF}$ and $\mathrm{Si}$, and $0.5 \mathrm{~mm}$ for the others). These thicknesses were chosen from the range calculations by the SRIM code [6]. The chamber was electrically insulated from the other experimental apparatuses to acquire the whole beam charge induced on the targets. In addition, a $125-\mu \mathrm{m}$-thick Mylar film was equipped with the chamber window at the forward directions. Neutrons emitted from the targets were detected by an EJ-301 liquid organic scintillator $(5.08-\mathrm{cm}$ diam. $\times 5.08-\mathrm{cm}$ length) placed at the distance of $2.4 \mathrm{~m}$ from the target position for forward 


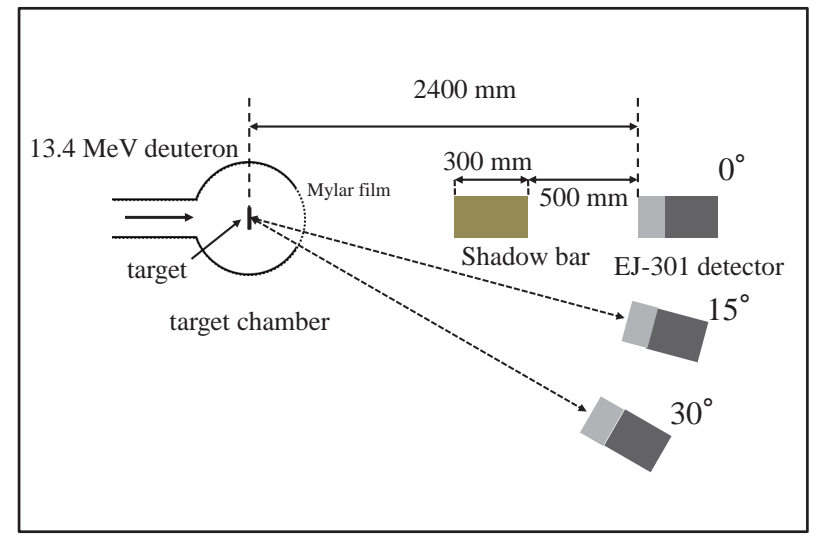

Figure 1. Schematic drawing of experimental setup. The iron shadow bar was placed only for background measurements.

emission angles (i.e., $0^{\circ}, 15^{\circ}$, and $30^{\circ}$ for $\mathrm{C}$ and $\mathrm{LiF}$, and $0^{\circ}$ for the others.) To estimate the contribution of neutrons scattered from floor and surrounding walls in the experimental room, additional background measurements were performed with an iron shadow bar (150-mm wide $\times$ 150 -mm high $\times 300$-mm thick) placed between the targets and detector.

\section{Data Analysis}

First, particle identification was performed by the conventional two-gate integration method because the EJ-301 detector is sensitive to gammas as well as neutrons. Figure 2 shows a two-dimensional plot of the total and slow components of the light output signals. Neutron events were clearly separated in the region of enough low light output. Next, the obtained light output spectra of neutron events per ADC channel were converted into those per light output units of electron equivalent. For this calibration, measurements of standard gamma sources ${ }^{137} \mathrm{Cs}(0.662 \mathrm{MeV})$ and ${ }^{60} \mathrm{Co}(1.17$ and $1.33 \mathrm{MeV})$ were performed. In addition, calibration in the higher light output region was performed by using gammas following ${ }^{12} \mathrm{C}(d, p){ }^{13} \mathrm{C}^{*}$ (3.09 and $\left.3.68 \mathrm{MeV}\right)$ and ${ }^{12} \mathrm{C}(d, d){ }^{12} \mathrm{C}^{*}$ $(4.44 \mathrm{MeV})$ reactions and neutrons emitted in ${ }^{7} \mathrm{Li}(d, n)^{8} \mathrm{Be}$ and ${ }^{12} \mathrm{C}(d, n){ }^{13} \mathrm{~N}$ reactions with maximum emission energies. Finally, neutron energy spectra were derived by an unfolding method using the response functions of the EJ-301 detector calculated by the SCINFUL-QMD code [7]. The unfolding of the measured light output spectra was performed by the FORIST code [8] based on the least-squares method.

Uncertainties in the experimental data are caused by the following three major factors: the geometry of the detector, the response functions of the detector, and the scattering of neutrons in the air. The first one was estimated to be $2 \%$ as the uncertainty contained in the determination of the solid angle of finite-size detector. The second one was estimated to be $17 \%$ from Ref. [2] considering the effect of aluminum housing of the detector and the difference of the response functions calculated

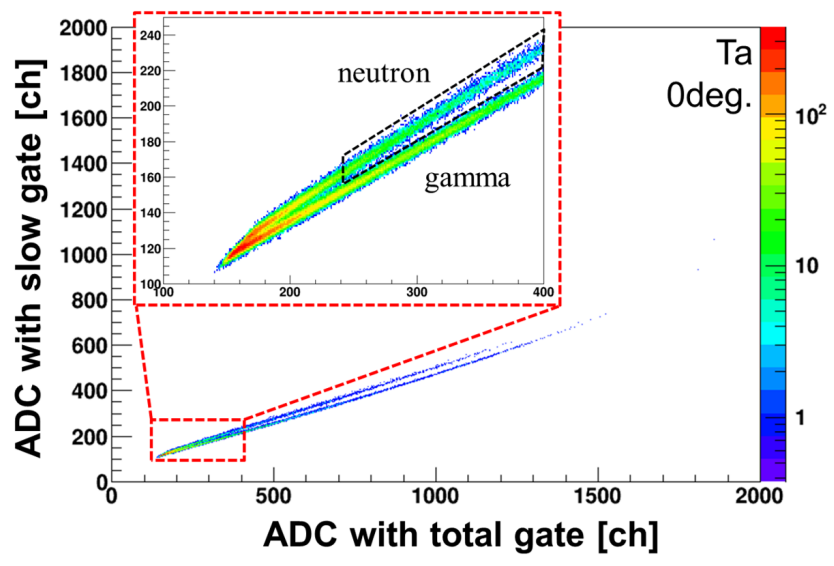

Figure 2. Two-dimensional plot of pulse integration with total (x-axis) and slow (y-axis) components for Ta target

by SCINFUL-QMD and CECIL [9] codes. The third one was estimated to be $2 \%$ on the basis of the neutron transport calculated with PHITS code. Contributions of the other sources such as the particle identification, beam integration, and dead time corrections were assumed to be less than $1 \%$.

\section{Results and Discussion}

\subsection{Neutron spectra from C and LiF targets}

The measured neutron spectra from $\mathrm{C}$ and $\mathrm{LiF}$ targets are shown in Fig. 3. Note that the error bars include only statistical uncertainties. Some bumps are observed around 5.5, 8.5, and $12 \mathrm{MeV}$ for $\mathrm{C}$, and 5.5, 15, and $26 \mathrm{MeV}$ for LiF. The first bump is caused by deuteron breakup as detailed in Sec. 4.4. The second and third bumps are due to proton-transfer reactions leading to the ground and first excited states in the residual nuclei. Table 1 shows

Table 1. Q-values of $(d, n)$ reactions in $\mathrm{LiF}$ and $\mathrm{C}$ targets

\begin{tabular}{|l|c|c|c|c|}
\hline reaction & \multicolumn{2}{|c|}{${ }^{7} \mathrm{Li}(d, n){ }^{8} \mathrm{Be}$} & \multicolumn{2}{c|}{${ }^{12} \mathrm{C}(d, n){ }^{13} \mathrm{~N}$} \\
\hline State of residual & g.s. & 1 st & g.s. & 1 st \\
\hline Q-value $[\mathrm{MeV}]$ & 15.0 & 3.60 & -0.280 & -3.78 \\
\hline
\end{tabular}
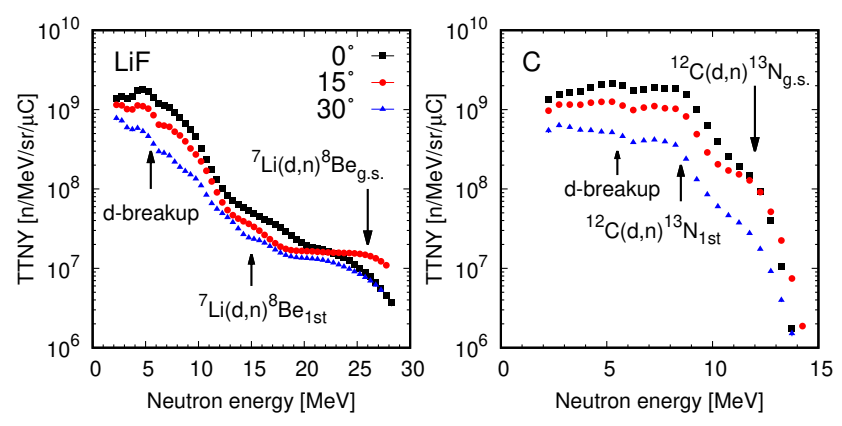

Figure 3. Double-differential neutron spectra from $\mathrm{LiF}$ (left) and $\mathrm{C}$ (right) targets at emission angles of $0^{\circ}, 15^{\circ}$, and $30^{\circ}$ 

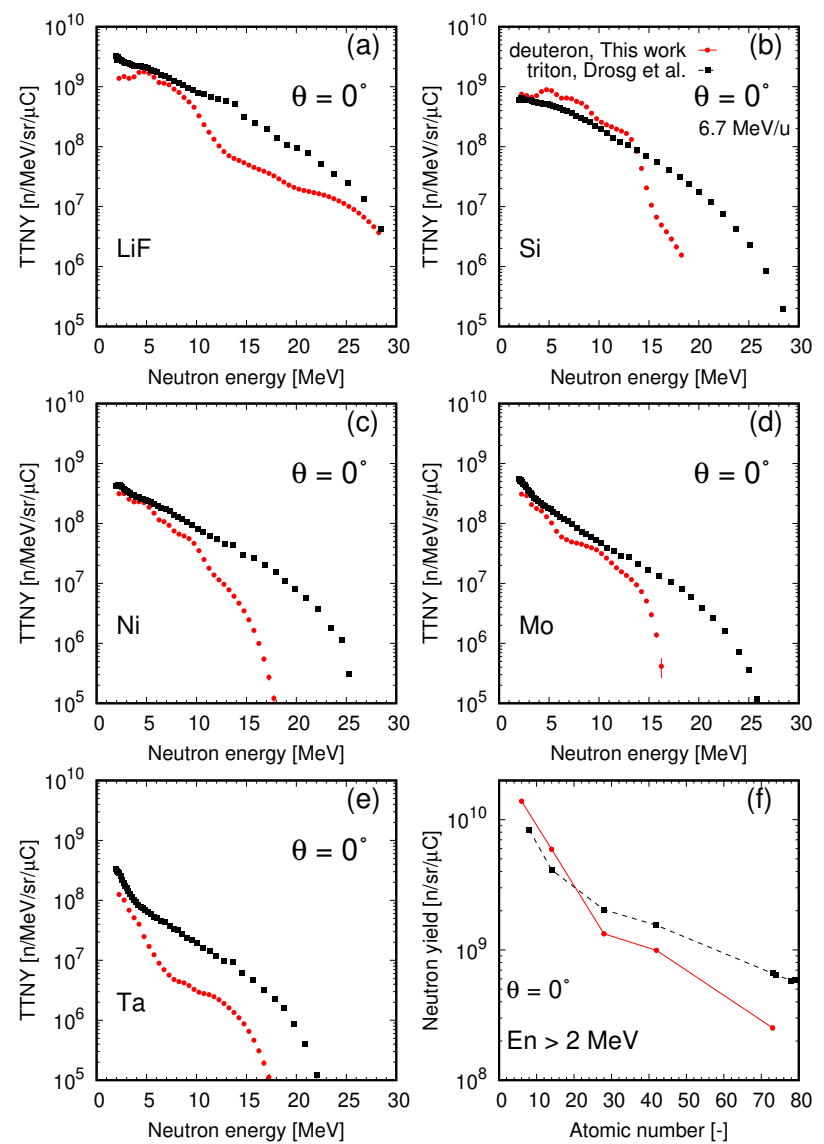

Figure 4. (a)-(e): Comparison of $(d, x n)$ and $(t, x n)$ spectra at an incident energy of $6.7 \mathrm{MeV} / \mathrm{u}$ from $\mathrm{LiF}, \mathrm{Si}, \mathrm{Ni}, \mathrm{Mo}$, and $\mathrm{Ta}$ targets at an emission angle of $0^{\circ}$. (f): Angle-differential neutron yield with emission energies $>2 \mathrm{MeV}$ versus atomic number of target elements. The solid (deuteron) and dashed (triton) lines are drawn to guide the eye.

the Q-values of proton-transfer reactions for $\mathrm{C}$ and $\mathrm{Li}$. It is apparent that the bumps correspond to the neutron emissions with each Q-value. The magnitudes of neutron spectra from both targets decrease rapidly as the emission angle increases. This tendency can be explained by the deuteron breakup since neutron emission by breakup is strongly forward-peaked.

\subsection{Comparison with $(t, x n)$ spectra}

Comparisons of the $(d, x n)$ and $(t, x n)$ spectra at an incident energy of $6.7 \mathrm{MeV} / \mathrm{u}$ are shown in Figs. 4a- 4e. In the $(d, x n)$ spectra, some characteristic bumps are observed for $\mathrm{LiF}$ and Si targets. These bumps are caused by deuteron breakup or proton-transfer reactions, as mentioned above, while those contributions are not observed for heavier targets. On the other hand, no specific structure is seen in any $(t, x n)$ spectra. This spectral difference may be due to the difference of neutron separation energies of the incident particles. That is, the separation energy of triton $\left(S_{n}=6.26 \mathrm{MeV}\right)$ is much larger than that of deuteron $\left(S_{n}=2.22 \mathrm{MeV}\right)$, and therefore, the contribution of projectile breakup should be smaller. Moreover, the triton breakup can be described by three-body kinematics
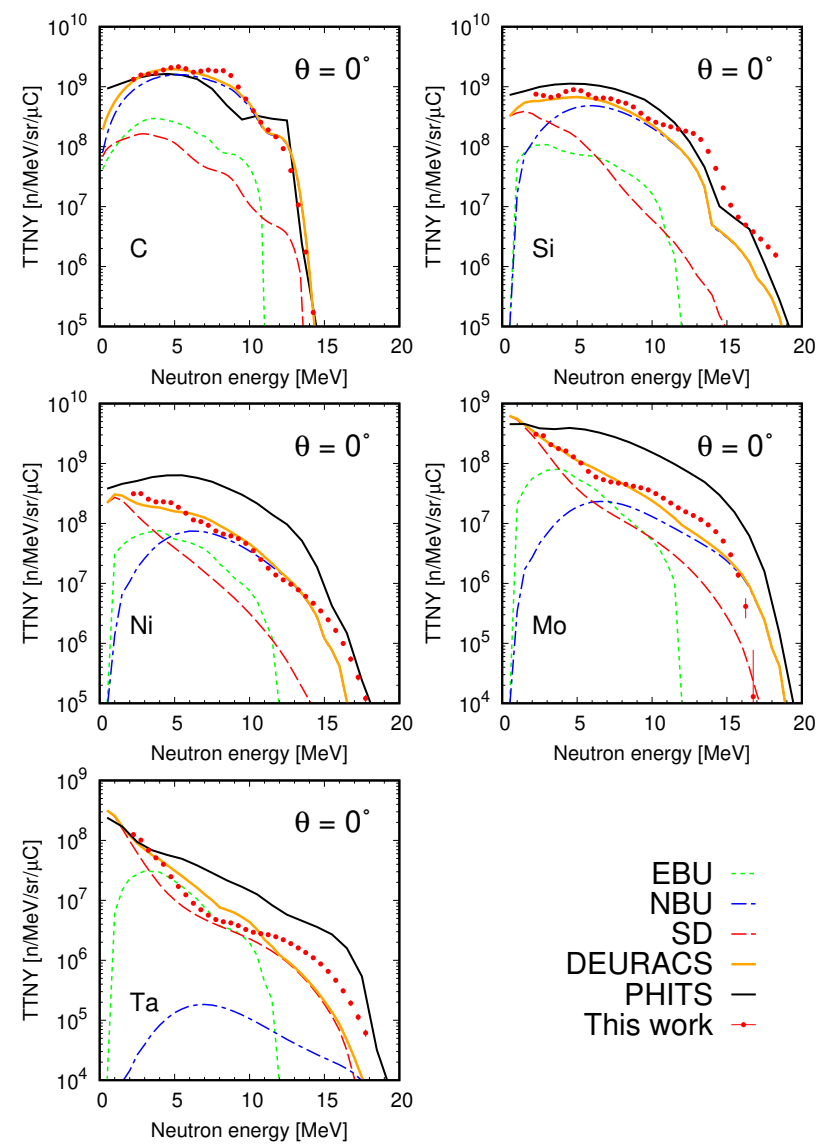

Figure 5. Experimental and calculated $(d, x n)$ spectra at an incident energy of $13.4 \mathrm{MeV}$. Calculations were performed with PHITS (black solid lines) and DEURACS (orange solid line) codes. Three components EBU (green dashed lines), NBU (blue dash-dotted lines), and SD (red dashed lines) calculated with DEURACS code are also shown.

$(t \rightarrow p+2 n)$, which leads to structureless spectra.

To compare neutron yields by deuteron and triton irradiations, the neutron spectra were integrated over neutron energies from $2 \mathrm{MeV}$ to the maximum emission energies. Fig. 4f shows the results as a function of target atomic number. The yields for the other elements measured in Ref. [5] are also plotted in the triton data. For both cases, the total neutron yields decrease exponentially with increasing atomic number. The deuteron irradiation produces more neutrons for low- $Z$ elements and decreases more rapidly in the high- $Z$ region than the triton incidence. Further detailed discussion for d-TTNYs will be given in Sec. 4.4 .

\subsection{Validation of PHITS calculations}

To benchmark the PHITS code for low-energy deuteron-induced reactions, its calculations are compared with the experimental $(d, x n)$ spectra. The intra-nuclear cascade of Liége (INCL) [10] and generalized evaporation model (GEM) [11] were utilized for dynamical process and subsequent evaporation process, respectively. Total reaction cross sections were calculated by using the Minomo-Washiyama-Ogata formula [12], which is 


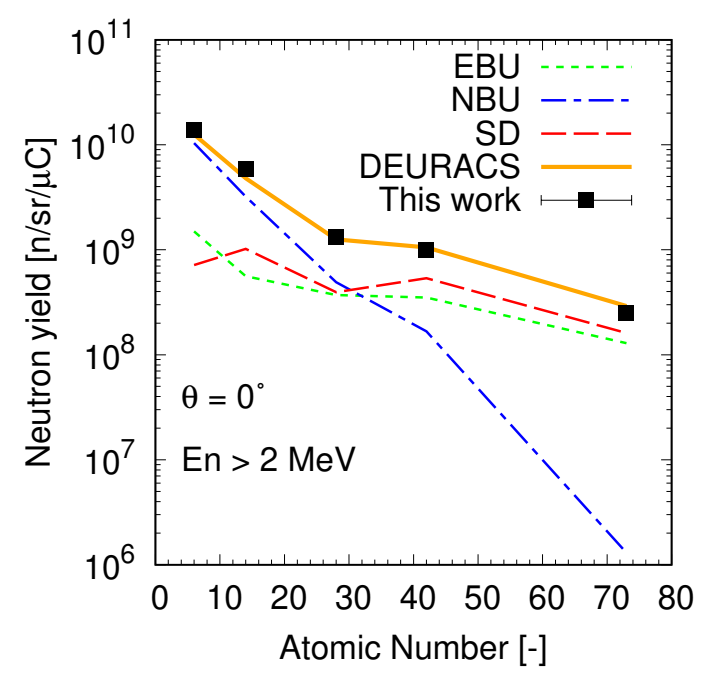

Figure 6. Angle-differential neutron yield with emission energy $>2 \mathrm{MeV}$ versus atomic number of target elements. Experimental (black squares) and calculated data by DEURACS (orange solid line) are shown along with each component.

dedicated for deuteron-nucleus reactions. The calculation results are shown in Fig. 5 (black lines). The PHITS calculation generally reproduces the spectral shapes for $\mathrm{C}$ and $\mathrm{Si}$ but overestimates the magnitudes for the heavier targets. Since the INCL was developed originally for intermediate energy reactions $>100 \mathrm{MeV} / \mathrm{u}$, this low energy may be beyond its applicability.

\subsection{Validation of DEURACS calculations}

In the DEURACS code, the elastic breakup (EBU) and non-elastic breakup (NBU) reactions are descried by the continuum discretized coupled channel theory and the Glauber model [13], respectively, and the statistical decay (SD) process is calculated with the CCONE code [14]. In addition, the distorted wave born approximation is employed for ${ }^{12} \mathrm{C}(d, n){ }^{13} \mathrm{~N}_{1 \text { st }}$ for $\mathrm{C}$ target. Fig. 5 shows the calculation results (orange lines) along with each component. DEURACS calculation reproduces the experimental spectra successfully. It was found that NBU is dominant for $\mathrm{C}$ and $\mathrm{Si}$ targets and makes the bumps around half the incident energy although its contribution is modest for the heavier targets. However, some discrepancies are seen between the calculation and experimental results. Bumps around $8.5 \mathrm{MeV}$ for $\mathrm{C}$ and around 13 and $17 \mathrm{MeV}$ for $\mathrm{Si}$ are not reproduced by DEURACS. These bumps are caused by proton-transfer reaction to the discrete levels of residues, which are not taken into account in this work.

To investigate the dependence of each component on target atomic number, the calculated spectra were integrated over neutron energy larger than $2 \mathrm{MeV}$ as done in Sec.4.2. The calculation result is shown in Fig.6. The contribution of NBU decrease drastically with increasing $Z$, leading to a steep falloff of neutron yield in the low- $Z$ region. In contrast, the contributions of EBU and SD decrease slightly in the wide $Z$ range, resulting in the gradual slope in the high- $Z$ region.

\section{Summary and Conclusions}

Double-differential neutron yields from six thick target materials ( $\mathrm{LiF}, \mathrm{C}, \mathrm{Si}, \mathrm{Ni}, \mathrm{Mo}$, and $\mathrm{Ta}$ ) bombarded by 13.4-MeV deuterons were measured. To investigate the difference in neutron production between deuterons and tritons at the same incident energy per nucleon, the measured $(d, x n)$ spectra were compared with the existing $(t, x n)$ data. Some bumps were observed in the deuteron case around half the incident energy, while no peak was observed for the triton case. In addition, the angle-differential neutron yields at $0^{\circ}$ were derived by integrating neutron spectra over neutron emission energy. The experimental neutron yields decrease exponentially as the target atomic number increases for both cases. The measured d-TTNY spectra were compared with the theoretical model calculations using PHITS and DEURACS. The PHITS calculation generally overestimates the experimental data for heavy targets, while DEURACS calculation agrees with experimental data fairly well.

This work was partially funded by ImPACT Program of Council for Science, Technology and Innovation (Cabinet Office, Government of Japan).

\section{References}

[1] A. Moeslang, V. Heinzel, H. Matsui, M. Sugimoto, Fusion Eng. and Des. 81, 863 (2006)

[2] S. Araki, Y. Watanabe, T. Kin, N. Shigyo, K. Sagara, Energy Procedia 71, 197 (2015)

[3] T. Sato, Y. Iwamoto, S. Hashimoto, T. Ogawa, T. Furuta, S. Abe, T. Kai, P.E. Tsai, N. Matsuda, H. Iwase et al., J. Nucl. Sci. Technol. 55, 684 (2018)

[4] S. Nakayama, H. Kouno, Y. Watanabe, O. Iwamoto, K. Ogata, Phys. Rev. C 94, 014618 (2016)

[5] M. Drosg, D.M. Drake, Nucl. Sci. Eng. 182, 256 (2016)

[6] J.F. Ziegler, M. Ziegler, J. Biersack, Nucl. Instrum. Meth. B 268, 1818 (2010), 19th International Conference on Ion Beam Analysis

[7] D. Satoh, T. Sato, A. Endo, Y. Yamaguchi, M. Takada, K. Ishibashi, J. Nucl. Sci. Technol. 43, 714 (2006)

[8] R. Johnson, D. Ingersoll, B. Wehring, J. Dorning, Nucl. Instrum. Meth. 145, 337 (1977)

[9] R. Cecil, B. Anderson, R. Madey, Nucl. Instrum. Meth. 161, 439 (1979)

[10] A. Boudard, J. Cugnon, J.C. David, S. Leray, D. Mancusi, Phys. Rev. C 87, 014606 (2013)

[11] S. Furihata, Nucl. Instrum. Meth. B 171, 251 (2000)

[12] K. Minomo, K. Washiyama, K. Ogata, J. Nucl. Sci. Technol. 54, 127 (2017)

[13] T. Ye, S. Hashimoto, Y. Watanabe, K. Ogata, M. Yahiro, Phys. Rev. C 84, 054606 (2011)

[14] O. Iwamoto, N. Iwamoto, S. Kunieda, F. Minato, K. Shibata, Nuclear Data Sheets 131, 259 (2016) 